\title{
Eagle Syndrome: A Diagnostic Challenge with a Novel Solution
}

\author{
Gaurav Chauhan, MD, Sandeep Kataria, MD, Jie-Xin Tang, MD, Vivek Loomba, MD, and \\ Aman Upadhyay, MD
}

Eagle Syndrome (ES), also referred to as styloid or stylohyoid syndrome, was first reported by otolaryngologist Watt W. Eagle in 1937. It refers to the myriad of signs and symptoms associated with an elongated styloid process or calcified stylohyoid ligament complex compressing on adjacent anatomical structures (1). The clinical spectrum of ES is comprised of nonspecific presentations such as facial pain, sore throat, otalgia, dysphagia, and or headaches (2). Patients suffering from ES are assigned various differential diagnosis and they may seek treatment at several different clinics such as otolaryngology, family practice, neurology, neurosurgery, psychiatry, or dentistry (3). We present a case report of a patient suffering from $\mathrm{ES}$, who was referred to the chronic pain clinic as a last resort from the otolaryngology clinic. The chronic pain team performed a successful fluoroscopicguided injection consisting of the steroid and local anesthetic mixture at the tip of the styloid process, leading to immediate and profound pain relief. This manuscript discusses the unique pathomechanics, diagnostic challenges and the solution offered by the authors.

\section{CASE REPORT}

We present a case report of a 57-year-old male, who consented to this case report to be published. The subject reported pain in right neck since last 3 years and sought multiple consults and assessments over the years with general practitioner, surgeon, dentist, and even a psychiatrist. The patient first presented

From : Henry Ford Health System, Detroit, MI

Author for correspondence: Gaurav Chauhan, MD

Address: Department of Anesthesiology, Henry Ford Health System,

Detroit, 1350 W. Bethune St., Apt 1607, Detroit, MI 48202

E-mail: Gchauha1@hfhs.org with sudden onset of pain, 3 years ago, in the right jaw with radiation to the right part of neck and entire face. At that point, he received emergency treatment for myocardial infarction but subsequent investigations including cardiac enzymes, electrocardiogram and stress echocardiogram came back negative for any acute cardiac pathology.

During the course of the next year, he presented multiple times to the emergency department and at his general practitioner's office with similar kinds of pain. He also reported a waxing and waning course of pain with severity of pain ranging from $2-8 / 10$ on the numeric rating scale. Over the course of 1 year he was assigned various diagnoses such as dental malocclusion, chronic otitis media, eustachian tube dysfunction, otalgia associated with chronic neck pain, etc., and received treatment ranging from a short-term course of antibiotics and opioids along with various non-steroidal anti-inflammatory (NSAIDs) drugs.

He was finally referred to the otolaryngology clinic where he was diagnosed with temporomandibular joint (TMJ) dysfunction along with myofascial pain syndrome. He was prescribed Gabapentin $300 \mathrm{mg} 3$ times a day, Topiramate $80 \mathrm{mg}$ twice a day, Methocarbamol $750 \mathrm{mg} 3$ times a day, Diclofenac $50 \mathrm{mg}$ twice a day and Desipramine $10 \mathrm{mg}$ nightly. Patient was also given a referral for physical therapy to stretch and strengthen his neck and jaw muscles.

The patient reported vivid dreams and unpleasant sensations after starting Gabapentin therapy. He was immediately advised to stop Gabapentin, which was substituted for Carbamazepine. The physician was unable to prescribe Pregabalin as it was not prescribed, and as it was not covered by the patient's health insurance. Over the course of a few 
weeks Desipramine was also discontinued, due to complaints of oral dryness and was substituted with Nortriptyline $25 \mathrm{mg}$ nightly. At 3 months follow-up, the patient reported that conservative management and physical therapy was successful in offering a mild decrease in the discomfort due to chronic pain but was not useful during the episodes of acute pain.

The otolaryngology team ruled out ES from the differential and the patient further underwent treatment for multitude of differentials including sinus infection, sensorineural hearing loss, neuropathic pain, etc. without any significant relief. At 3 years from the initial presentation and at 2 years from presentation at otolaryngologist's office, after multiple cycles of diagnostic and therapeutic failures, patient reported that he was getting frustrated and was beginning to feel depressed due to his chronic pain.

He further reported that the pain was adversely affecting his quality of life. In lieu of his suboptimal symptom control he was referred to the pain clinic and to a chronic pain psychologist. In the pain clinic, on examination, the patient reported no change in character of his right-sided, anterolateral and upper neck pain which was exacerbated by yawning or turning the head on the opposite side and was associated with dysphagia, odynophagia and globus sensation in the throat. He did not endorse any trauma, nor had he any history of a procedure or surgery on the right side of the neck. There were no obvious bony processes observed or perceived on palpation of the neck but patient-reported pain on palpation of the right peritonsillar fossa.

The patient had been taking Diclofenac $50 \mathrm{mg}$ twice a day, Methocarbamol $750 \mathrm{mg} 3$ times a day, along with Nortriptyline $25 \mathrm{mg}$ nightly at this point, which seemed to offer some degree of benefit. The lateral $x$-ray of neck and computerized tomographic (CT) scan revealed a bilateral elongated styloid process (left side $-6 \mathrm{~cm}$ and right side $-5 \mathrm{~cm}$ ) which was more pronounced on the asymptomatic side (left) (Figs. 1 and 2).

On the basis of the physical exam, radiographic evidence of elongated styloid processes, protracted clinical course and vague symptomatology, a possibility of ES was reconsidered, primarily as a diagnosis of exclusion. Consequently, the authors planned to perform a fluoroscopically-guided injection of a mixture of local anesthetic and non-particulate steroid at the tip of the styloid process on the affected side as a diagnostic and therapeutic option.

The risks, benefits and alternatives were explained in detail to the patient and he consented to the procedure. The right-sided styloid process was identified by fluoroscopy in the lateral projection. For added safety, the approximate depth of the styloid process was extrapolated from the previous CT images. Under aseptic precautions, using a 25 gauge, 11/2-inch needle, the right-sided styloid process was reached in fluoroscopic projection view and was confirmed with the anteroposterior view. A $0.5 \mathrm{~mL}$ iodinated contrast was injected to confirm position and rule out the abnormal spread (Fig.3). A $3 \mathrm{~mL}$ solution comprising $2 \mathrm{~mL}$ of $0.5 \%$ Bupivacaine and $4 \mathrm{mg}$ of Dexamethasone, was injected in the intended space.

The patient reported excellent and an almost immediate relief of his pain. The pain relief lasted for 9 days and the patient further commented that this was the only thing that had helped his disabling condition in the past 3 years.

These new findings were communicated to the otolaryngology team and the patient was subsequently scheduled for excision of the right styloid process via an extra-oral approach. During the surgical procedure a solution of $1 \%$ lidocaine with 1:200000 epinephrine was injected into the left neck 2 fingerbreadths below the angle of the mandible.

A standard neck incision was made 2 fingerbreadths below the angle of the mandible from mastoid to the edge of the mandible. The dissection was performed along the sternocleidomastoid muscle (SCM). The posterior auricular nerve was identified and spared and the anterior border of the SCM was dissected free from underlying soft tissue. A blunt dissection was then used to identify the spinal accessory nerve and this was dissected free from the overlying tissue. The posterior belly of the digastric was identified and dissection was then performed medially deep to the digastric muscle.

The stylomastoid muscle was identified and dissected off at the styloid process at the angle of the mandible 

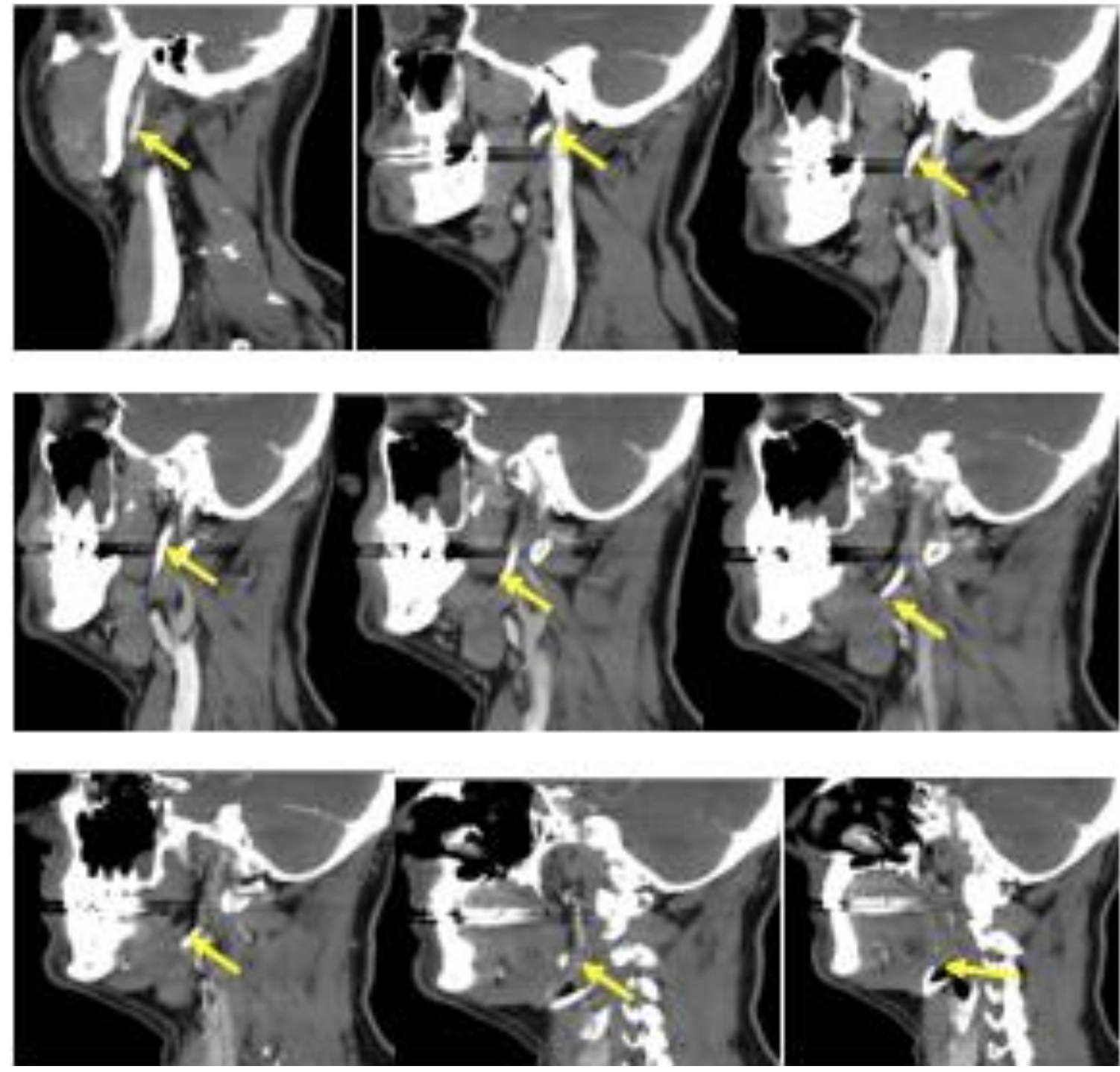

Fig. 1. CT Scan of Cervical soft tissue - Sagittal view, serial views from right to left. Yellow arrow depicting the subsegmental ossification of both styloid processes. The ossified ligaments run all the way to the lesser cornua of the hyoid bone bilaterally, indenting the base of the tongue, right vallecular space, and running in between the external and internal carotid arteries. The right demonstrates more ossification and calcification compared to the left. There is no clear-cut attachment to the temporal bone of stylohyoid ligament.

using a freer elevator and a periosteal elevator. The styloid process was then stripped of the tissues in all directions. A bone cutter was then used to cut the distal portion of the styloid process. The bone was removed. A rongeur was used to round the proximal cut edge of the styloid process. The incision was then re-approximated with 3-0 vicryl and the superficial skin was closed with DERMABOND adhesive (Ethicon US, LLC). The patient reported excellent pain relief with remission of symptoms at 6 months follow-up after the surgery. He further reported that he has stopped taking Diclofenac and requested for Nortriptyline to be tapered off. 


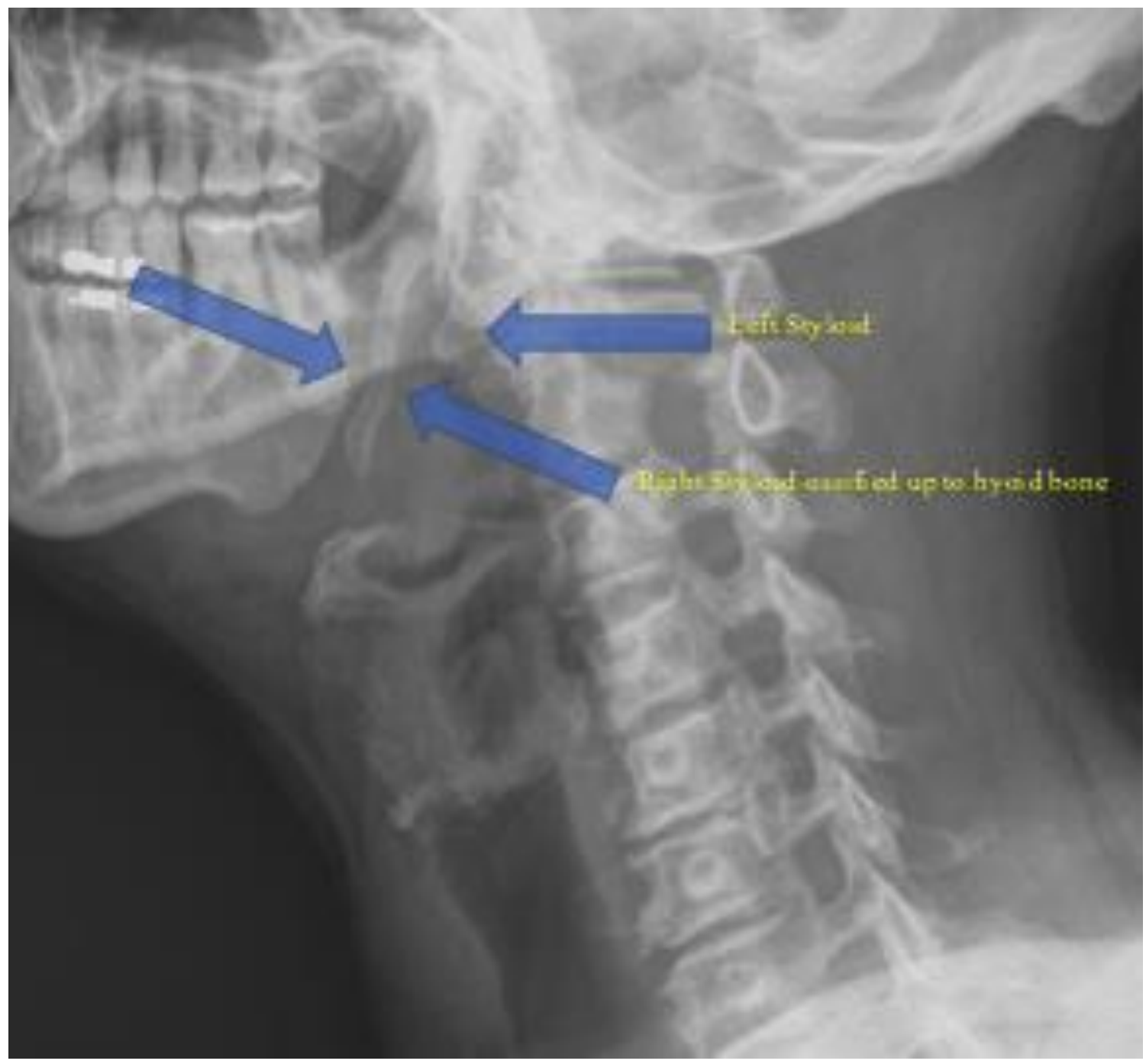

Fig. 2. Lateral x-ray of cervical region: Bilateral segmental ossification of the stylohyoid processes and ligaments. Right styloid is more ossified than the left styloid process.

\section{DISCUSSION}

The embryonic origin of the styloid process is from endochondral ossification of the Reichert's cartilage of the second pharyngeal arch (4). It is an elongated, conical, variably tapered extension of the inferior surface (petrous part) of the temporal bone that projects downwards and forwards from the inferior surface of the temporal bone. The styloid process lies between the internal and external carotid arteries and is bounded posteriorly by the mastoid process and medially by tonsillar fossa. Its proximal part, known as tympanohyal is encased by the tympanic aspect of the temporal bone and its distal part, also known as stylohyal, serves as an insertion point for the stylohyoid and stylomandibular ligaments and styloglossus, stylohyoid and stylopharyngeus muscles. The stylohyoid ligament connects the apex of the styloid process and the lesser horn of the hyoid bone, and the stylomandibular ligament extends from the styloid process to the parotideomasseteric fascia between the mastoid process and the mandible $(2,5)$. 

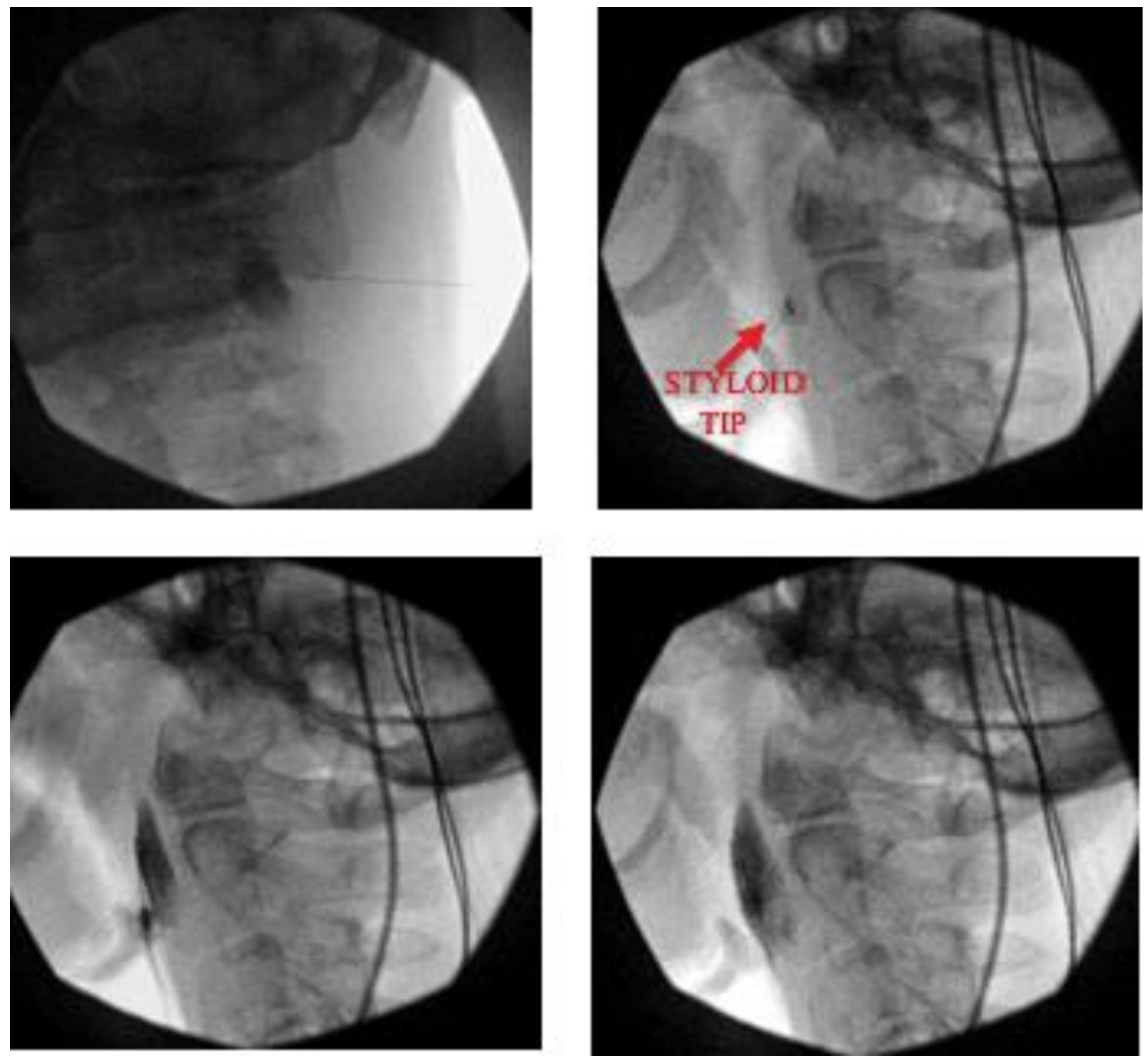

Fig. 3. A sagittal fluoroscopic view of base of skull and neck depicting the tip of styloid process localized by injection of Isovue contrast dye.

The length of a typical styloid process ranges from 1.52 to $4.77 \mathrm{~cm}$, however, a $3 \mathrm{~cm}$ or longer process is usually considered anomalous (6). The incidence of an elongated styloid process and the calcified stylohyoid ligament is reported to be $3.3 \%$ and $18.2 \%$, respectively but only $1-5 \%$ of the subjects with radiographic evidence develop symptoms $(6,7)$. Due to the lack of epidemiological studies the current literature is ambivalent in regards to the sexual predilection of ES; however, the symptoms are more common in females and the patients are usually older than 30 years of age (4).
The mechanics of ES may include direct compression of adjacent structures by an elongated styloid process due to degenerative and inflammatory changes in the cartilaginous precursors (insertion tendinosis) of the stylohyoid ligament (8). The etiology of the late onset of symptoms includes reactive hyperemia, metaplasia due to abnormal healing and fibrosis following recurrent throat infections, scar tissue formation following trauma (induced or iatrogenic e.g. tonsillectomy) and age-related changes such as cervical disc degeneration which may lead to the 
shortening of cervical vertebrae and altering the direction of the styloid process which can further cause distortion of cranial nerves or cranial nerve endings in structures adjacent to the styloid process (8). Ectopic ossification or mineralization of the stylohyoid makes it more vulnerable to fractures. The cycles of recurrent fractures and subsequent ossification further fuels the proliferation of granulation tissue, which impinges on adjacent structures. The constellation of symptoms in a patient suffering from ES is independent of the degree of calcification or size of styloid process as chronic impingement of surrounding tissues in the pharyngeal mucosa, either directly due to styloid process or as a result of peri-styloid inflammation, sets up a cascade of events leading to further chronic inflammation, edema, granuloma formation, adhesions and fibrosis leading to further irritation and pain.

The neural elements most commonly involved are the mandibular branch of the trigeminal nerve, chorda tympani branch of VII nerve, IX and X nerves $(3,6)$. The diagnosis is guided by the clinical history, physical exam and corroborated by the radiological evidence and a high index of suspicion. The characteristic symptoms may include recurrent throat pain associated with foreign body sensation or dysphagia along with radiation to ipsilateral ear or jaw and may be triggered or exacerbated by head rotation, tongue movement or during deglutition. A careful transpharyngeal palpation may demonstrate a bony projection in the tonsillar fossa and may reproduce the characteristic pain, which may be referred to the ear, face or head $(3,7)$. The patients frequently complain of a foreign body sensation in the throat along with dysphagia, odynophagia, dysgeusia or alterations in taste and a change in the character of their voice. Infrequently, patients have also reported migraine-like headaches and occipital neuralgias as a manifestation of referred pain $(7,9)$. In more rare instances, an enlarged styloid process may also impinge upon the carotid sinus of the internal carotid artery, leading to the transient ischemic attack, dizziness and vagal mediated cardiac inhibition, which can prove to be fatal (10-12).

Various imaging techniques such as panoramic radiography, lateral cephalometry, Towne projection film, or CT scan, may be employed to aid the diagnosis. A
CT scan or CT guided 3-dimensional reconstruction can yield the exact spatial orientation, the anatomic variance of styloid process and its association with the surrounding tissues $(9,10)$. Despite the radiological evidence of an elongated styloid process, we used a mixture of local anesthetic and steroid solution and injected at the tip of styloid process under fluoroscopic guidance to further affirm the diagnosis. There are case reports in which authors have injected local anesthetic in the tonsillar fossa or employed stellate ganglion blocks to mitigate pain; however, these procedures are technically challenging and are not bereft of serious side effects (13). The fluoroscopic injection technique is relatively straightforward, and offers a more precise means of diagnosis as it offers the advantage of using less of a volume of local anesthetic injected.

Conservative management should be the first line of treatment as it has a high success rate $(80 \%$ of cases) and can lead to can lead to complete resolution of symptoms. Conservative management of ES includes multimodal analgesics such as non-steroidal anti-inflammatory drugs, which may have added benefit to reduce local inflammation, gabapentinoids and tricyclic antidepressants for the neuropathic components of the pain $(14,15)$. If conservative management fails or in the presence of acute severity of symptoms, an en-bloc excision, either extra-oral or endoscopically guided, of the styloid process offers definitive management (11). After the dissection of the right styloid process the subject had gradual decrease and complete resolution of his chronic throat pain.

In conclusion, the pathognomonic signs and symptoms of ES are vague and share a multitude of etiologies making it a diagnostic challenge. In our case, there was a delay of 3 years from onset of symptoms to the definitive management, during which the patient underwent a great deal of stress and psychological trauma. A multidisciplinary collaboration between chronic pain, otolaryngology, and psychiatry, was required to identify, diagnose and treat the rare diagnosis of ES. This is the first instance of the utilization of a simple, fluoroscopically guided injection technique for confirmatory diagnosis of this rare condition. 
Eagle Syndrome: A Diagnostic Challenge with a Novel Solution

\section{REFERENCES}

1. Dunn-Ryznyk LR, Kelly CW. Eagle syndrome: A rare cause of dysphagia and head and neck pain. JAAPA 2010; 23:28-48.

2. Fusco DJ, Asteraki S, Spetzler RF. Eagle's syndrome: Embryology, anatomy, and clinical management. Acta Neurochir (Wien) 2012; 154:1119-1126.

3. Ghosh LM, Dubey SP. The syndrome of elongated styloid process. Auris Nasus Larynx 1999; 26:169-175.

4. Badhey A, Jategaonkar A, Anglin Kovacs AJ, Kadakia S, De Deyn PP, Ducic Y, Schantz S, Shin E. Eagle syndrome: A comprehensive review. Clin Neurol Neurosurg 2017; 159:34-38.

5. Valerio CS, Peyneau PD, de Sousa AC, Cardoso FO, de Oliveira DR, Taitson PF, Manzi FR. Tylohyoid syndrome: Surgical approach. J Craniofac Surg 2012; 23:e138-e140.

6. Balcioglu, HA, Kilic, C, Akyol, M, Ozan, H, Kokten, G. Length of the styloid process and anatomical implications for Eagle's syndrome. Folia Morphol 2009; 68:265-270.

7. Colby CC, Del Gaudio JM. Stylohyoid complex syndrome: A new diagnostic classification. Arch Otolaryngol Head Neck Surg 2011; 137:248-252.

8. Correll, RW, Jensen, JL, Taylor, JB, Rhyne, RR. Mineralization of the stylohyoid-stylomandibular ligament complex. A ra- diographic incidence study. Oral Surg Oral Med Oral Path 1979; 48:286-291.

9. Murtagh RD, Caracciolo JT, Fernandez G. CT findings associated with Eagle syndrome. Am J Neuroradiol 2001; 22:1401-1402.

10. Montalbetti L, Ferrandi D, Pergami P, Savoldi F. Elongated styloid process and Eagle's syndrome. Cephalalgia 1995; 15:80-93.

11. Ceylan A, Köybaşioğlu A, Celenk F, Yilmaz O, Uslu. Surgical treatment of elongated styloid process: experience of 61 cases. Skull Base 2008; 18:289-295.

12. Ruwanpura, PR, Abeygunasekera, AM, Tikiri, GK. Sudden unexpected death probably due to Eagle's syndrome: A case report. Med. Sci. Law 2008; 48:350-353.

13. Nickel, J, Sonnenburg, M, Scheufler, O, Anderson, R. Eagle's syndrome: Diagnostic imaging and therapy. Rontgenpraxis 2003; 55:108-113.

14. Taheri A, Firouzi-Marani S, Khoshbin M. Nonsurgical treatment of stylohyoid (Eagle) syndrome: A case report. J Korean Assoc Oral Maxillofac Surg 2014; 40:246-249.

15. Han MK, Kim DW, Yang JY. Non-surgical treatment of Eagle's Syndrome - A case report. Korean J Pain 2013; 26:169-172. 
